\title{
SPACES OF TRANSITIVE INTERVAL MAPS
}

\author{
SERGIǏ KOLYADA, MICHAŁ MISIUREWICZ, AND L'UBOMÍR SNOHA
}

\begin{abstract}
On a compact real interval, the spaces of all transitive maps, all piecewise monotone transitive maps and all piecewise linear transitive maps are considered with the uniform metric. It is proved that they are contractible and uniformly locally arcwise connected. Then the spaces of all piecewise monotone transitive maps with given number of pieces as well as various unions of such spaces are considered and their connectedness properties are studied.
\end{abstract}

\section{INTRODUCTION AND MAIN RESULTS}

In this paper we investigate topological properties of the space of all transitive maps of a compact interval to itself and its subspaces.

When mathematicians are considering various classes of functions with a natural topology, often one of the first questions that comes to their mind is about the topological properties of those spaces. While those questions have been answered long ago in Mathematical Analysis and Topology, it seems that they even have not been asked in Dynamical Systems. As far as we were able to establish, there are no papers on topological properties of spaces of maps that have some specific dynamical properties. The only exception is the paper of Farrell and Gogolev [2] about the spaces of Anosov diffeomorphisms, that was being written at the same time as ours (and completely independently of ours). Let us stress that we mean properties of given functional spaces, not relations between various spaces (like, for instance, that one space is a dense or residual subset of another one).

The area of Dynamical Systems where one investigates dynamical properties that can be described in topological terms is called Topological Dynamics. Investigating topological properties of spaces of maps that can be described in dynamical terms is in a sense the opposite idea. Therefore we propose to call this area Dynamical Topology. It is on the boundary between Dynamical Systems and Topology, but, in our opinion, much closer to Dynamical Systems, because most of the tools that can be used there are from dynamics.

Let $X$ be a compact metric space and let $f: X \rightarrow X$ be continuous. The dynamical system $(X, f)$ is called topologically transitive (or just transitive) if for every pair of nonempty open sets $U$ and $V$ in $X$ there is a nonnegative integer $n$ such that $f^{n}(U) \cap V \neq \emptyset$. If the space $X$ has no isolated points, this is equivalent to the

Date: August 6, 2013.

2010 Mathematics Subject Classification. Primary 37E05; Secondary 37B05, 54H20.

Key words and phrases. Transitive interval map, $n$-modal map, contractible space.

The first author was supported by the John von Neumann Visiting Professorship (Zentrum Mathematik, Technische Universität München); he acknowledges the hospitality of the Zentrum. The third author was supported by VEGA grant 1/0978/11 and by the Slovak Research and Development Agency under the contract No. APVV-0134-10. 
existence of a point $x \in X$ whose orbit $\left\{x, f(x), \ldots, f^{n}(x), \ldots\right\}$ is dense in $X$. Consequently, a topologically transitive dynamical system cannot be decomposed into two disjoint sets with nonempty interiors which do not interact under the transformation. In particular, transitivity is an ingredient of several definitions of chaos. For more information on topological transitivity see, e.g., [4] and references there.

Here our space is a compact interval $I$. Clearly, it does not matter which interval we choose, so to fix notation (and to fix the length of the interval) we make the standard choice $I=[0,1]$. Maximal intervals of monotonicity of a continuous map $f: I \rightarrow I$ are called laps of $f$. By transitivity, two laps can intersect at most at the common endpoint. If $f$ is differentiable at $x$, then by the slope of $f$ at $x$ we mean $\left|f^{\prime}(x)\right|$.

As we mentioned, this is mainly work in dynamics. The main difficulty is to construct families of transitive maps. How can one show that those maps are transitive? Here are some ideas that we are using.

- A map conjugate to a transitive map is transitive.

- A piecewise linear map that has large slopes and laps with long images is often transitive.

- Even if those images are not long, if the map is additionally close to a transitive map, it is often transitive.

We will use the following notation. When we say "piecewise", we mean that there are finitely many pieces.

- $I=[0,1]$;

- id - the identity map from $I$ to $I$;

- $\mathcal{C}$ - the space of all continuous maps from $I$ to $I$;

- $\mathcal{H}$ - the space of all homeomorphisms from $I$ onto $I$;

- $\mathcal{H}^{+}$- the subspace of all orientation preserving homeomorphisms of $\mathcal{H}$;

- $\mathcal{T}$ - the space of all topologically transitive maps of $\mathcal{C}$;

- $\mathcal{T}_{\mathrm{PM}}$ - the space of all piecewise monotone maps of $\mathcal{T}$;

- $\mathcal{T}_{\mathrm{PL}}$ - the space of all piecewise linear maps of $\mathcal{T}$;

- $\mathcal{T}_{n}$ - the space of all elements of $\mathcal{T}_{\mathrm{PM}}$ of modality $n$;

- $\mathcal{T}_{n}^{+}$- the space of all elements of $\mathcal{T}_{n}$ increasing on the first lap;

- $\mathcal{T}_{n}^{-}$- the space of all elements of $\mathcal{T}_{n}$ decreasing on the first lap.

All those spaces are considered with the $C^{0}$ metric $d$ :

$$
d(f, g)=\sup _{x \in I}|f(x)-g(x)| .
$$

We will write $\mathcal{T}_{n}^{*}$ for one of the spaces $\mathcal{T}_{n}^{+}, \mathcal{T}_{n}^{-}$(that is, $*=+$ or $*=-$ ). For an interval $J$ we will denote its length by $|J|$. We will refer to the left and right endpoints of a closed interval $J$ as $\min J$ and $\max J$, respectively.

In this paper we investigate the spaces $\mathcal{T}, \mathcal{T}_{\mathrm{PM}}, \mathcal{T}_{\mathrm{PL}}, \mathcal{T}_{n}$, and the unions of $\mathcal{T}_{n}$ 's for various $n$ 's. Notice that the space $\mathcal{T}$ is clearly nowhere dense (and not closed) in the space $\mathcal{C}$, and even in the space of all surjective maps from $\mathcal{C}$. The main results of the paper are the following three theorems.

Theorem A. The spaces $\mathcal{T}, \mathcal{T}_{\mathrm{PM}}$ and $\mathcal{T}_{\mathrm{PL}}$ are contractible (in particular, they are arcwise connected) and locally connected (in fact, they are uniformly locally arcwise connected). 
Theorem B. For every $m>n \geq 1$, the space $\mathcal{T}_{n} \cup \mathcal{T}_{m}$ is arcwise connected.

Theorem C. For every $n \geq 1$ there is a loop in $\mathcal{T}_{n} \cup \mathcal{T}_{n+1}$, which is not contractible in $\mathcal{T}_{n} \cup \mathcal{T}_{n+1}$.

The paper is organized as follows. In Section 2 we investigate spaces $\mathcal{T}, \mathcal{T}_{\mathrm{PM}}$ and $\mathcal{T}_{\mathrm{PL}}$, and prove Theorem A (see Theorem 2.5 and Proposition 2.7). In Section 3 we consider smaller spaces, namely $\mathcal{T}_{n}$, and the unions of $\mathcal{T}_{n}$ 's for various $n$ 's. In particular, we prove Theorem B (see Corollary 3.11 and Theorem 3.15) and Theorem C (see Theorem 3.19).

\section{SPACES WITH NO RESTRICTIONS ON MODALITY}

We start with a construction that will be used several times in this section.

Given a closed interval $K$, we define box maps $\xi: K \rightarrow \mathbb{R}$, that depend on five parameters:

- $a_{l}$ - the value at the left endpoint of $K$,

- $a_{r}$ - the value at the right endpoint of $K$,

- $a_{b}$ - the bottom of the box,

- $a_{t}$ - the top of the box,

- $a_{s}$ - the slope multiplier.

By the box we will understand the rectangle $K \times\left[a_{b}, a_{t}\right]$, so we will assume $a_{t}>a_{b}$. The image of $K$ will be equal to $\left[a_{b}, a_{t}\right]$, so we will assume that $a_{l}, a_{r} \in\left[a_{b}, a_{t}\right]$. Moreover, we want $a_{s} \geq 20$. The map $\xi$ will be piecewise linear with constant slope, and this slope will be $a_{s}\left(a_{t}-a_{b}\right) /|K|$.

We make the laps of $\xi$ as large as possible, that is, the graph of $\xi$ goes to the top, then to the bottom, etc., of the box. Since the slope of $\xi$ is constant, this graph can be viewed as a billiard trajectory in the box (see Figure 1). We make this construction both from the left and right. When moving from an end of $K$, the graph on the first lap goes up, so $\xi$ is increasing on the leftmost lap and decreasing on the rightmost one. In the case when this is impossible, that is, when $a_{l}=a_{t}$ or $a_{r}=a_{t}$, we do what we can, that is, the graph on the first lap goes down. The left and right graphs (billiard trajectories) have to meet eventually. We choose this meeting point $m$ to be on the fifth decreasing lap from the left (see again Figure 1). If the left and right graphs coincide, then there is no meeting point (at least no well defined one).

Lemma 2.1. The map $\xi$ depends continuously on parameters $a_{l}, a_{r}, a_{b}, a_{t}$ and $a_{s}$ (jointly).

Proof. We shall prove that locally, in a small neighborhood of a given $\xi_{0}$, the map $\xi$ is Lipschitz continuous as the function of each of the parameters. This will establish the local uniform continuity of $\xi$ as the function of all parameters jointly.

As the function of each of $a_{l}$ and $a_{r}$, the map $\xi$ is Lipschitz continuous with constant 1 . This follows from the fact that moving $a_{l}$ (or $a_{r}$ ) results in moving the unfolding of the corresponding billiard trajectory by the same amount, and the folding operation is Lipschitz continuous with constant 1. Moreover, in the interval between the meeting points of the unperturbed and perturbed maps $\xi$, the distance between the billiard trajectories is even smaller than the perturbation (if there is no meeting point for one or both of them, the situation is even simpler). 


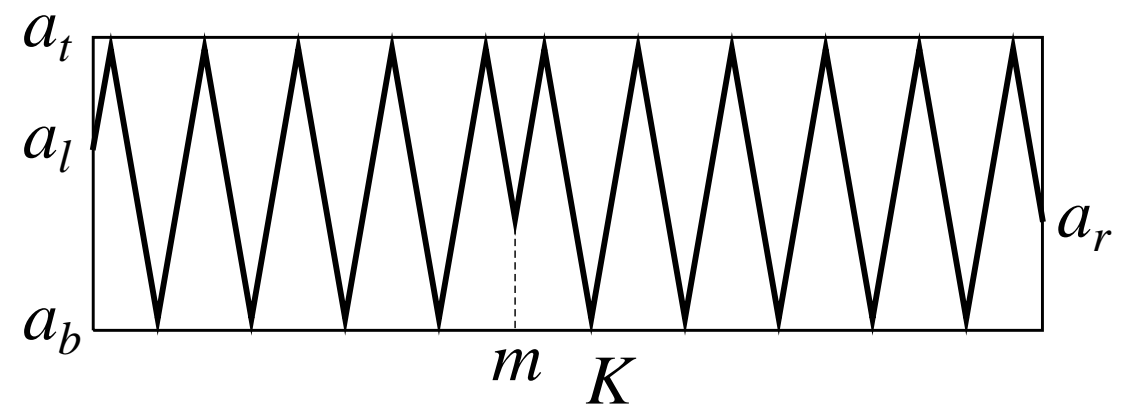

Figure 1. The graph of $\xi$ on $K$, with $a_{s}=20$. Note that in the applications the interval $\left[a_{b}, a_{t}\right]$ is usually much longer than $K$.

Moving $a_{b}$ (or $a_{t}$ ) results in an increase of the distance between the billiard trajectories by 2 times the perturbation size of $a_{b}$ (or $a_{t}$ ) at every reflection from the bottom (or the top) of the box. Since the number of reflections is uniformly bounded in a small neighborhood of $\xi_{0}$, it follows that $\xi$ is locally Lipschitz continuous as a function of each of $a_{b}$ and $a_{t}$.

Moving $a_{s}$ by $\varepsilon$ results in moving the slope of $\xi$ by $\varepsilon\left(a_{t}-a_{b}\right) /|K|$, so the distance between the unperturbed and perturbed $\xi$ is at most $\varepsilon\left(a_{t}-a_{b}\right)$. This proves that $\xi$ is locally Lipschitz continuous as a function of $a_{s}$.

Now, using the box maps, we will construct a certain family of maps

$$
\left\{g_{f, t}: f \in \mathcal{T}, t \in[0,1]\right\} .
$$

This family will have in particular the following properties:

(a) $g_{f, 0}=f$ for every $f$,

(b) $g_{f, t}$ depends continuously on $f$ and $t$ (jointly),

(c) $g_{f, t} \in \mathcal{T}_{\mathrm{PL}}$ for every $f \in \mathcal{T}$ and $t>0$.

Since our family has to satisfy (a), we set $g_{f, 0}=f$. Thus, we have to deal only with $t>0$. To simplify notation, fix $f \in \mathcal{T}$ and $t>0$ and denote $g_{f, t}$ by $g$.

Let $s$ be the largest nonnegative integer such that $s t<1$. For $i=0,1, \ldots, s-1$ we set $I_{i}=[i t,(i+1) t]$ and call those intervals normal partition intervals. Each of them has length $t$. Additionally, we set $I_{s}=[s t, 1]$ and call this interval the short partition interval (except when its length is $t$; then it is normal). Its length is less than or equal to $t$. In particular, if $t=1$ then $s=0$, and the interval $I_{0}=I$ is normal.

Now for each $i$ we set $\alpha_{i}=\max \left\{\left|I_{i}\right|,\left|f\left(I_{i}\right)\right|\right\}$, and $J_{i}=\left[a_{i, b}, a_{i, t}\right]$, where $a_{i, b}=$ $\max \left\{0, \min f\left(I_{i}\right)-4 \alpha_{i}\right\}$ and $a_{i, b}=\min \left\{1, \max f\left(I_{i}\right)+4 \alpha_{i}\right\}$ (see Figure 2).

Observe that $f\left(I_{i}\right) \subset J_{i}$ and

$$
\text { either } J_{i}=I \text { or }\left|J_{i}\right| \geq 4 \alpha_{i} \text {. }
$$

On each $I_{i}$ we define $g$ as the box map with $K=I_{i}, a_{l}=f\left(\min I_{i}\right), a_{r}=f\left(\max I_{i}\right)$, $a_{b}=a_{i, b}, a_{t}=a_{i, t}$, and $a_{s}=20$. Note that by the definition, $\alpha_{i} \geq\left|I_{i}\right|$ and so, by equation (1), either $\left|J_{i}\right|=|I| \geq\left|I_{i}\right|$ or $\left|J_{i}\right| \geq 4 \alpha_{i} \geq 4\left|I_{i}\right|$. In any case, $\left|J_{i}\right| /\left|I_{i}\right| \geq 1$ and thus the slope of $g$ on $I_{i}$ is at least 20 .

Clearly, the map $g$ defined in this way is piecewise linear and continuous. We have to prove (b) and (c). We begin with (b). 


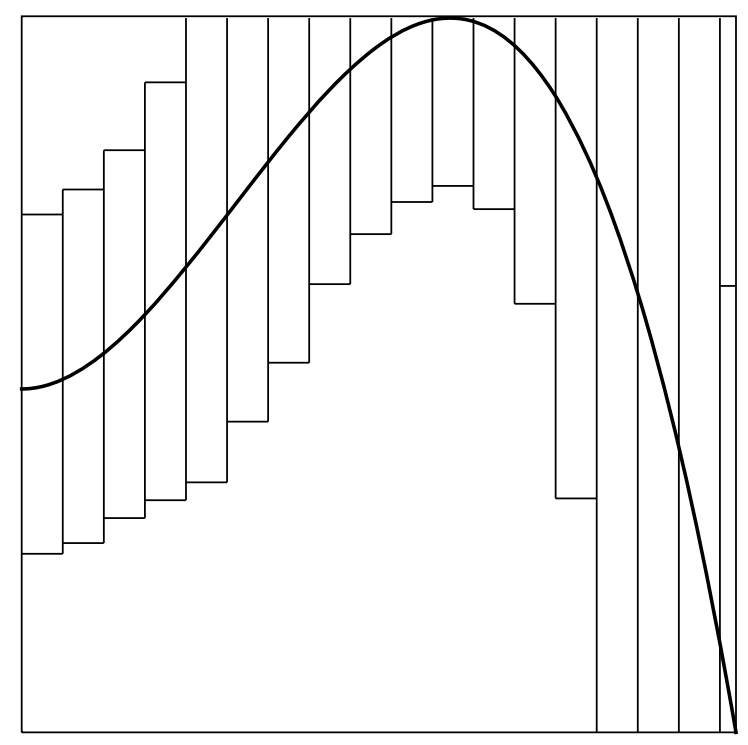

Figure 2. Boxes $I_{i} \times J_{i}$.

Lemma 2.2. The map $g_{f, t}$ depends continuously on $f$ and $t$ (jointly).

Proof. We start by proving continuity of the map $(f, t) \mapsto g_{f, t}$ at the points $(f, 0)$. Fix $f$ and $d_{0}>0$. We are going to show that $d\left(g_{\tilde{f}, \tilde{t}}, g_{f, 0}\right)=d\left(g_{\tilde{f}, \tilde{t}}, f\right)<d_{0}$ whenever $\widetilde{t}>0$ is sufficiently small and $\widetilde{f}$ is sufficiently close to $f$.

Consider the partition of $I$, as defined above, corresponding to $\widetilde{t}>0$. It consists of the intervals $I_{0}, \ldots, I_{s}$ (the integer $s$ depends on $\widetilde{t}$. Since $f$ is uniformly continuous, for all sufficiently small $\widetilde{t}>0$ both $\left|I_{i}\right|$ and $\left|f\left(I_{i}\right)\right|$ are shorter than $d_{0} / 27$ for all $i$. Let $d(\widetilde{f}, f)<d_{0} / 27$ and let $K_{i}$ be the convex hull of $f\left(I_{i}\right) \cup \widetilde{f}\left(I_{i}\right)$. Of course, $\left|K_{i}\right|<d_{0} / 9$ and so $\widetilde{\alpha}_{i}:=\max \left\{\left|I_{i}\right|,\left|K_{i}\right|\right\}<d_{0} / 9$. Put $\widetilde{J}_{i}=I \cap\left[\min K_{i}-4 \widetilde{\alpha}_{i}, \max K_{i}+4 \widetilde{\alpha}_{i}\right]$. Then $\left|\widetilde{J}_{i}\right|<d_{0}$, and both the graph of $f$ and the graph of $g_{\widetilde{f}, \tilde{t}}$ are subsets of $\bigcup_{i=0, \ldots, s} I_{i} \times \widetilde{J}_{i}$. It follows that $d\left(g_{\tilde{f}, \tilde{t}}, g_{f, 0}\right)=d\left(g_{\tilde{f}, \tilde{t}}, f\right)<d_{0}$.

Now we prove continuity of the map $(f, t) \mapsto g_{f, t}$ at the points $(f, t)$, where $t>0$ is not of the form $1 / k, k \in \mathbb{N}$. Fix $f$ and $t>0$ that is not of the form $1 / k, k \in \mathbb{N}$. When we say that something is as small as we want or that two objects are as close to each other as we want, we mean that this happens if $\tilde{f}$ is close enough to $f$ and $\widetilde{t}$ is close enough to $t$.

The last of the intervals $I_{0}, \ldots, I_{s}$ that are assigned to $t$ is short. Therefore, if $\widetilde{t}>0$ is close enough to $t$ (and from now on we will consider only such $\widetilde{t}$ ), we have the same number of intervals $\widetilde{I}_{0}, \ldots, \widetilde{I}_{s}$ assigned to $\widetilde{t}$ and still $\widetilde{I}_{s}$ is short. The set

$$
I \backslash \bigcup_{i=0}^{s}\left(I_{i} \cap \widetilde{I}_{i}\right)
$$

consists of at most $s$ intervals (more precisely, it is empty if $\widetilde{t}=t$, and otherwise it consists of exactly $s$ intervals). 
Those intervals are as short as we want and the values of $g_{f, t}$ and $g_{\widetilde{f}, \tilde{t}}$ on those intervals are as close to the values of $f$ as we want (remember that at the partition points for $t$, the maps $f$ and $g_{f, t}$ are equal and at the partition points for $\tilde{t}$, the maps $\widetilde{f}$ and $g_{\widetilde{f}, \tilde{t}}$ are equal). Therefore, it is sufficient to show that for each $i \in\{0, \ldots, s\}$ the distance of maps $g_{f, t}$ and $g_{\widetilde{f}, \tilde{t}}$ restricted to $Q_{i}:=I_{i} \cap \widetilde{I}_{i}$ is as small as we want. However, on $Q_{i}$ both $g_{f, t}$ and $g_{\tilde{f}, \tilde{t}}$ are box maps with corresponding parameters as close to each other as we want, so by Lemma $2.1 g_{f, t}$ and $g_{\widetilde{f}, \tilde{t}}$ are as close to each other as we want. The only possible exception is when $f\left(\min I_{i}\right)=1$ and $\tilde{t}>t$ (or $f\left(\max I_{i}\right)=1$ and $\left.\tilde{t}<t\right)$; then on $Q_{i}$ the map $g_{f, t}$ is decreasing on the first lap (or increasing on the last lap), so formally it is not a box map. However, then we can virtually extend both $g_{f, t}$ and $g_{\widetilde{f}, \tilde{t}}$ to a slightly larger interval, so that they are both box maps, and then we can use Lemma 2.1.

Finally, we show continuity of the map $(f, t) \mapsto g_{f, t}$ at the points $(f, 1 / k), k \in \mathbb{N}$. Fix $f$ and $t=1 / k, k \in \mathbb{N}$. If $\tilde{t}$ is close enough to $t$ and $\tilde{t} \geq t$, then the family of intervals $I_{0}, \ldots, I_{k-1}$ which corresponds to $t$ and the family of intervals $\widetilde{I}_{0}, \ldots, \widetilde{I}_{k-1}$ which corresponds to $\tilde{t}$, have the same number of intervals. The proof is then the same as in the preceding case, when $t \neq 1 / k, k \in \mathbb{N}$.

If $\widetilde{t}$ is close enough to $t$ and $\tilde{t}<t$, the family $\widetilde{I}_{0}, \ldots, \widetilde{I}_{k-1}, \widetilde{I}_{k}$ has one element more than the family $I_{0}, \ldots, I_{k-1}$, since a short interval $\widetilde{I}_{k}$ appears. For $i<k$ we proceed as before. Moreover, we observe that the new interval $\widetilde{I}_{k}$ is as short as we want and the values of $g_{\widetilde{f}, \tilde{t}}$ on it are as close as we want to $f(\max I)=g_{f, t}(\max I)$. This completes the proof.

Now we want to prove (c).

Lemma 2.3. Assume that $f \in \mathcal{T}$ and $t>0$. Then the map $g=g_{f, t}$ is piecewise linear and transitive.

Proof. Since $g$ is piecewise linear, we need to prove only its transitivity. Let $L \subset I$ be an interval. We claim that if both $|L|<2 t$ and $|g(L)|<2 t$ then either $|g(L)| \geq 2|L|$ or $g(L)=I$. To prove it, assume that $|L|<2 t$ and $|g(L)|<2 t$. If $L$ is contained in the union of 4 laps of $g$ then, since the slope of $g$ is at least 16 (in fact, it is at least 20 , but we need only 16), we have $|g(L)| \geq 4|L|$. If $L$ is not contained in the union of 4 laps of $g$ then there is a lap $K$ of $g$ such that $K \subset L \cap I_{i}$ and $g(K)=J_{i}$ for some $i$. If $I_{i}$ is a normal partition interval, then we have $|g(L)| \geq\left|J_{i}\right| \geq 4\left|I_{i}\right|=4 t>2|L|$, or $g(L)=I$ (see (1)). If $I_{i}$ is the short partition interval (that is, $i=s$ ), then, as above, $|g(L)| \geq\left|J_{s}\right| \geq 4\left|I_{s}\right|$ or $g(L)=I$. Thus, either we are done, or $4\left|I_{s}\right| \leq 2|L|$, and then there is a subinterval $L^{\prime} \subset L$ with $\left|L^{\prime}\right| \geq|L| / 2$, disjoint from $I_{s}$. In the latter case, by the same argument as earlier, either $\left|g\left(L^{\prime}\right)\right| \geq 4\left|L^{\prime}\right| \geq 2|L|$, or $\left|g\left(L^{\prime}\right)\right| \geq 4 t>2|L|$, or $g\left(L^{\prime}\right)=I$. This completes the proof of the claim.

Let $\mathcal{A}$ be the family of all intervals which are unions of partition intervals, at least one of them being normal. We will show that if $L \in \mathcal{A}$ then there is $K \in \mathcal{A}$ such that $f(L) \subset K \subset g(L)$. Using this by induction we get $f^{k}(L) \subset g^{k}(L)$ for all $k$. Since $f$ is transitive, the $f$-trajectory of $L$ is dense, so the $g$-trajectory is also dense. Then the $g$-trajectory of every interval is dense. In fact, if $M$ is any interval, then from the claim it follows that there is $n$ such that $\left|g^{n}(M)\right| \geq 2 t$ or $g^{n}(M)=I$. However, if 
$\left|g^{n}(M)\right| \geq 2 t$, then $g^{n}(M)$ contains a normal partition interval, and normal partition intervals belong to $\mathcal{A}$.

Thus, it remains to show that if $L \in \mathcal{A}$ then there is $K \in \mathcal{A}$ such that $f(L) \subset$ $K \subset g(L)$. Assume first that $L$ is a normal partition interval $I_{i}$. Then $g(L)=J_{i}$ and $J_{i}$ is the union of 3 intervals: $f\left(I_{i}\right)=f(L)$ and two adjacent intervals of length at least $4 \alpha_{i} \geq 4\left|I_{i}\right|=4 t$ each (more precisely, the intersections of two such intervals with $I$ - therefore the two adjacent intervals may in fact be shorter than $4 t$ or even degenerate).

However, in those lateral intervals there must be points of the partition, and this allows us to choose $K$. In a general case, we write $L$ as the union of normal partition intervals $L_{j}, j=1, \ldots, m$ and perhaps the short partition interval $I_{s}$. For each of $L_{j}$ we get the corresponding interval $K_{j} \in \mathcal{A}$ with $f\left(L_{j}\right) \subset K_{j} \subset g\left(L_{j}\right)$ and then we take $K=\bigcup_{j=1}^{m} K_{j}$. It is connected because $\bigcup_{j=1}^{m} f\left(L_{j}\right)$ is connected. If $I_{s}$ is involved then either $\left|f\left(I_{s}\right)\right| \geq t / 2$ and the situation is the same as for the normal partition interval (each of the two adjacent intervals has length at least $4 \alpha_{s} \geq 4\left|f\left(I_{s}\right)\right| \geq 2 t$ and so it contains a point of the partition), or $\left|f\left(I_{s}\right)\right|<t / 2$ and then for $j$ such that $L_{j}=I_{s-1}$ we can choose $K_{j}$ containing $f\left(I_{s}\right)$. This completes the proof.

Remark 2.4. The above proof still works if we make some modifications in the definition of $g_{f, t}$. In particular, we can replace intervals $J_{i}$ by larger intervals and we can increase the slope multipliers $a_{s}$ (independently for every $i$ ). Indeed, both operations keep slopes large enough, so that the first part of the proof works, and keep intervals $J_{i}$ large enough, so that the second part of the proof works.

Recall that a topological space $X$ is contractible if the identity map on $X$ is nullhomotopic, i.e. if it is homotopic to some constant map. All the homotopy groups of a contractible space are trivial. Every contractible space is pathwise connected. A space $X$ is pathwise connected if for any two points $x$ and $y$ in $X$, there is a continuous map $f: I \rightarrow X$ such that $f(0)=x$ and $f(1)=y$. Such a map $f$ (as well as its range $f(I)$, when confusion is not possible) is called a path from $x$ to $y$. We call $X$ arcwise connected if for any two points $x$ and $y$ in $X$, there is a homeomorphism $f: I \rightarrow X$ onto its image such that $f(0)=x$ and $f(1)=y$. The map $f$ (as well as its range) s called an arc from $x$ to $y$. Every Hausdorff path from $x$ to $y$ contains an arc from $x$ to $y$. Thus a Hausdorff space is pathwise connected if and only if it is arcwise connected.

Theorem 2.5. The spaces $\mathcal{T}, \mathcal{T}_{\mathrm{PM}}$ and $\mathcal{T}_{\mathrm{PL}}$ are contractible. In particular, they are arcwise connected.

Proof. Recall that $g_{f, 0}=f$. The structure of the maps $g_{f, 1}$ is very simple. They are box maps with $K=[0,1], a_{b}=0, a_{t}=1$ and $a_{s}=20$. They depend only on 2 parameters: $a_{l}=f(0)$ and $a_{r}=f(1)$. This parametrization gives a homeomorphism of the space $Z$ of those maps with a square. Our family $g_{f, t}$ can be treated as a homotopy joining the identity selfmap of the space $\mathcal{T}$ ( or $\mathcal{T}_{\mathrm{PM}}$, or $\mathcal{T}_{\mathrm{PL}}$ ) with the map of $\mathcal{T}$ (or $\mathcal{T}_{\mathrm{PM}}$, or $\mathcal{T}_{\mathrm{PL}}$ ) into $Z$. This proves that the spaces $\mathcal{T}, \mathcal{T}_{\mathrm{PM}}$ and $\mathcal{T}_{\mathrm{PL}}$ are contractible.

Lemma 2.6. Any $f, \tilde{f} \in \mathcal{T}$ can be joined by an arc $A$ of diameter smaller than or equal to $5 d(f, \tilde{f})$ such that $A \backslash\{f, \widetilde{f}\} \subset \mathcal{T}_{\mathrm{PL}}$. 
Proof. Set $\varepsilon=d(f, \widetilde{f})$. By Lemma 2.2 and uniform continuity of $f$ and $\tilde{f}$, there is $t_{0}>0$ such that

(i) $d\left(g_{f, t}, f\right) \leq \varepsilon / 2$ and $d\left(g_{\widetilde{f}, t}, \widetilde{f}\right) \leq \varepsilon / 2$ for all $t \in\left[0, t_{0}\right]$, and

(ii) all intervals $J_{i}$ and $\widetilde{J}_{i}$ used in the definitions of $g:=g_{f, t_{0}}$ and $\widetilde{g}:=g_{\widetilde{f}, t_{0}}$ have lengths at most $\varepsilon$.

Let $K_{i}$ be the smallest interval containing $J_{i}$ and $\widetilde{J}_{i}$. Then, if we denote by $I_{0}, \ldots, I_{s}$ the partition intervals corresponding to $t_{0}$, the graphs of $f, \widetilde{f}, g$ and $\widetilde{g}$ are subsets of the union of the boxes $I_{i} \times K_{i}, i=0,1, \ldots, s$. By (ii), the length of each $K_{i}$ is at $\operatorname{most}\left|J_{i}\right|+d(f, \widetilde{f})+\left|\widetilde{J}_{i}\right| \leq 3 \varepsilon$.

To prove the lemma, it is sufficient to find a path $P$ from $f$ to $\widetilde{f}$ with required properties. It will be the union of four paths.

The first path is a path from $f$ to $g$, given by $\left[0, t_{0}\right] \ni t \mapsto g_{f, t}$. Similarly, the fourth path is $\left[0, t_{0}\right] \ni t \mapsto g_{\widetilde{f}, t}$, followed backward, i.e. from $\widetilde{g}$ to $\widetilde{f}$. By (i), each of these two paths has diameter at most $\varepsilon$. The second path is obtained by moving linearly on each $I_{i}$, for the corresponding box maps, $a_{b}$ from $\min J_{i}$ to $\min K_{i}$ and $a_{t}$ from $\max J_{i}$ to $\max K_{i}$. The third path is similar, but we move linearly four parameters: $a_{b}$ from $\min K_{i}$ to $\min \widetilde{J}_{i}, a_{t}$ from $\max K_{i}$ to $\max \widetilde{J}_{i}, a_{l}$ from $f\left(\min I_{i}\right)$ to $\widetilde{f}\left(\min I_{i}\right)$, and $a_{r}$ from $f\left(\max I_{i}\right)$ to $\widetilde{f}\left(\max I_{i}\right)$. By Lemma 2.1 , both paths are continuous. Their concatenation joins $g$ with $\widetilde{g}$ and lies in the union of the boxes $I_{i} \times K_{i}, i=0,1, \ldots, s$. Since $\left|K_{i}\right| \leq 3 \varepsilon$, it has diameter at most $3 \varepsilon$ and so diameter of the whole $P$ is at most $\varepsilon+3 \varepsilon+\varepsilon=5 \varepsilon$. By Lemma 2.3 and Remark 2.4, all maps from the path $P$ are transitive. Since they all, except perhaps $f$ and $\widetilde{f}$, are also piecewise linear, the proof is complete.

A metric space $X$ with metric $d$ is uniformly locally arcwise connected if for any $\varepsilon>0$ there is a $\delta>0$ such that whenever $0<d(x, y)<\delta$, then $x$ and $y$ are joined by an arc of diameter $<\varepsilon$, see e.g. [3, p. 129]. The space $X=((0,1] \times\{0\}) \cup$ $(\{1,1 / 2,1 / 3, \ldots\} \times[0,1])$ is arcwise connected and locally arcwise connected but not uniformly locally arcwise connected.

Given a space $X, A \subseteq X$ and $p \in X \backslash A$, we say that $p$ is arcwise accessible from the set $A$ if for every $a \in A$ there is an arc from $a$ to $p$ lying in $A \cup\{p\}$, see e.g. [8, p. 111] or [3, p. 119]).

Proposition 2.7. $\quad$ (a) The spaces $\mathcal{T}, \mathcal{T}_{\mathrm{PM}}$ and $\mathcal{T}_{\mathrm{PL}}$ are uniformly locally arcwise connected. In particular, they are locally connected.

(b) Every map from $\mathcal{T}$ is arcwise accessible from the set $\mathcal{T}_{\mathrm{PL}}$.

Proof. (a) follows from Lemma 2.6. To get (b), fix $f \in \mathcal{T}$, choose $f \neq \tilde{f} \in \mathcal{T}$, use Lemma 2.6 and the fact that, by Theorem $2.5, \mathcal{T}_{\mathrm{PL}}$ is arcwise connected.

Recall that the metric spaces $\mathcal{T}, \mathcal{T}_{\mathrm{PM}}$ and $\mathcal{T}_{\mathrm{PL}}$ are separable. A separable metric space is said to be locally infinite-dimensional if every nonempty open subset of this space is infinite-dimensional.

Proposition 2.8. The spaces $\mathcal{T}, \mathcal{T}_{\mathrm{PM}}$ and $\mathcal{T}_{\mathrm{PL}}$ are locally infinite-dimensional.

Proof. Since $\mathcal{T}_{\mathrm{PL}}$ is dense both in $\mathcal{T}$ and $\mathcal{T}_{\mathrm{PM}}$, it is sufficient to prove that $\mathcal{T}_{\mathrm{PL}}$ is locally infinite-dimensional. Fix $f \in \mathcal{T}_{\mathrm{PL}}$ and $\eta>0$. Let $U$ be the $\eta$-neighborhood 
of $f$ in $\mathcal{T}_{\mathrm{PL}}$. To prove that $U$ is infinite-dimensional, we fix any $n \in \mathbb{N}$ and we prove that $U$ has dimension at least $n$. In fact, we show that $U$ contains a subset homeomorphic with the $n$-dimensional compact cube $[20,21]^{n}$. Choose small $t>0$ such that $n t \leq 1$ and all the rectangles $I_{i} \times J_{i}$ (see Fig. 2) involved in the definition of $g_{f, t}$ lie in that subset of $I \times I$ which corresponds to $U$ (i.e., $f(x)-\eta<y<f(x)+\eta$ whenever $[x, y] \in I_{i} \times J_{i}$ for some $i$ ). Note that there are at least $n$ partition intervals $I_{0}, \ldots, I_{n-1}$.

For every $c_{0}, \ldots c_{n-1} \in[20,21]$ we can modify the definition of $g_{f, t}$ by taking the slope multiplier $a_{s}$ equal to $c_{i}$, instead of 20, on $I_{i}$. Such a modification yields a transitive piecewise linear map by Lemma 2.3 and Remark 2.4. The map $\Phi:[20,21]^{n} \rightarrow$ $\mathcal{T}_{\mathrm{PL}}$ that we obtain, is continuous by Lemma 2.1. Clearly, it is one-to-one. Since $[20,21]^{n}$ is compact, $\Phi$ is a homeomorphism onto its image. By the construction, $\Phi\left([20,21]^{n}\right) \subset U$. This completes the proof.

\section{Spaces With Restrictions on modality}

We start with a lemma, which is well known, but which is quite difficult to find in the literature. Since the proof is very simple, we include it.

Lemma 3.1. The following three maps are continuous:

(a) the map $g \mapsto g \circ f$ from $\mathcal{C}$ to $\mathcal{C}$, for a given $f \in \mathcal{H}$;

(b) the map $g \mapsto f \circ g$ from $\mathcal{C}$ to $\mathcal{C}$, for a given $f \in \mathcal{H}$;

(c) the map $g \mapsto g^{-1}$ from $\mathcal{H}$ to $\mathcal{H}$.

Proof. Fix $f \in \mathcal{H}$. If $g, h \in \mathcal{C}$ then

$$
d(g \circ f, h \circ f)=d(g, h),
$$

because the range of $f$ is $I$. This proves (a).

The map $f$ is uniformly continuous, so for every $\varepsilon>0$ there is $\delta>0$ such that if $|x-y|<\delta$ then $|f(x)-f(y)|<\varepsilon$. Thus,

$$
\forall_{\varepsilon>0} \exists_{\delta>0} d(g, h)<\delta \Rightarrow d(f \circ g, f \circ h)<\varepsilon .
$$

This proves (b).

Let us now fix $g \in \mathcal{H}$. By (3) applied to $f=g^{-1}$ we get

$$
\forall_{\varepsilon>0} \exists_{\delta>0} d(g, h)<\delta \Rightarrow d\left(\mathrm{id}, g^{-1} \circ h\right)<\varepsilon .
$$

Now we apply (2) for $f=h^{-1}$, with $g$ replaced by id and $h$ replaced by $g^{-1} \circ h$. We get

$$
\forall_{\varepsilon>0} \exists_{\delta>0} d(g, h)<\delta \Rightarrow d\left(h^{-1}, g^{-1}\right)<\varepsilon
$$

This proves (c).

We will say that $f, g \in \mathcal{C}$ are positively conjugate if they are conjugate via a map from $\mathcal{H}^{+}$.

Lemma 3.2. If $\mathcal{A}$ is one of the classes $\mathcal{T}_{\mathrm{PM}}, \mathcal{T}_{n}, \mathcal{T}_{n}^{*}$ and $f, g \in \mathcal{A}$ are positively conjugate then there is an arc in $\mathcal{A}$ joining $f$ with $g$. 
Proof. Let $h \in \mathcal{H}^{+}$be a conjugacy between $f$ and $g$, that is, $g=h^{-1} \circ f \circ h$. For $t \in[0,1]$ set

$$
h_{t}(x)=t h(x)+(1-t) x .
$$

Since a convex combination of increasing functions is increasing, all maps $h_{t}$ belong to $\mathcal{H}^{+}$. We have $h_{0}=$ id and $h_{1}=h$.

Set $f_{t}=h_{t}^{-1} \circ f \circ h_{t}$ for $t \in[0,1]$. Each $f_{t}$ is positively conjugate to $f$, so it is in the same class $\mathcal{A}$ as $f$. By Lemma 3.1, the map $h_{t} \mapsto f_{t}$ is continuous, so since the map $t \mapsto h_{t}$ is also continuous, $\left\{f_{t}: t \in[0,1]\right\}$ is an arc. We have $f_{0}=f$ and $f_{1}=g$, and the proof is complete.

We will be using the theorem of W. Parry ([7], see also [6] and [1]).

Theorem 3.3. Every transitive piecewise monotone interval map is positively conjugate to a map of constant slope.

Note that in the original statement there is just "conjugate" instead of "positively conjugate." However, if the conjugacy is orientation reversing, we may apply afterward the conjugacy via the map $x \mapsto 1-x$. The resulting map will have still constant slope, and the composition of the two conjugacies will be a positive one.

In what follows we will say that $t \in I$ is a turning point of $f \in \mathcal{T}_{\mathrm{PM}}$ if it is an interior point of $I$ at which $f$ has a local extremum.

Lemma 3.4. For a map $f \in \mathcal{T}_{m}^{*}$, its turning point $c$, and a neighborhood $U$ of $c$, there exists an arc in $\mathcal{T}_{m}^{*}$, whose one endpoint is $f$ and the other endpoint is a map $g$ with $g(x)=f(x)$ for $x \notin U$ and such that $g(c)=1$ if $f$ has a local maximum at $c$ and $g(c)=0$ if $f$ has a local minimum at $c$. The same holds if $c$ is an endpoint of $I$.

Proof. We will write the proof for the case when $c$ is a turning point. The proof in the case when $c$ is an endpoint of $I$ is practically the same. A slight difference occurs in one place; we will comment on this.

We may assume that $f$ has a local maximum at $c$; otherwise we consider a map conjugate with $f$ via $x \mapsto 1-x$. Moreover, by Theorem 3.3 we may assume that $f$ has constant slope. Indeed, after we construct an arc for the map of the constant slope, we transport it to the original setting via the inverse to our conjugacy $h$. That is, we transport via $h^{-1}$ the point $c$, the neighborhood $U$, and all maps from the arc. What we get is again an arc, because if $h \in \mathcal{H}$ then the map $\varphi \mapsto h^{-1} \circ \varphi \circ h$ from $\mathcal{C}$ to $\mathcal{C}$ is surjective, continuous by Lemma 3.1 and has continuous inverse $\psi \mapsto h \circ \psi \circ h^{-1}$. Thus, in the rest of the proof we assume that $f$ has constant slope $\lambda$. Since $f$ is transitive, $\lambda>1$.

In order to facilitate invoking various statements, we will mark them with capital letters.

Take $k$ such that $\lambda^{k}>4$, and let $\varepsilon$ be the length of the shortest lap of $f^{k}$. Note that every lap of $f^{k}$ is contained in some lap of $f$, so the lengths of all laps of $f$ are at least $\varepsilon$.

(A) For any interval $L$ of length less than $\varepsilon$ we have $\left|f^{k}(L)\right|>2|L|$. Indeed, if $|L|<\varepsilon$ then $L$ contains at most one turning point of $f^{k}$, so $\left|f^{k}(L)\right| /|L| \geq \lambda^{k} / 2>2$.

(B) If $L$ is an interval of length at least $\varepsilon$ then $\left|f^{n}(L)\right|>\varepsilon / \lambda^{k}$ for every $n$. To prove this, suppose that $|L| \geq \varepsilon$, but $\left|f^{n}(L)\right| \leq \varepsilon / \lambda^{k}<\varepsilon / 4$ for some $n \geq 1$. We may assume 


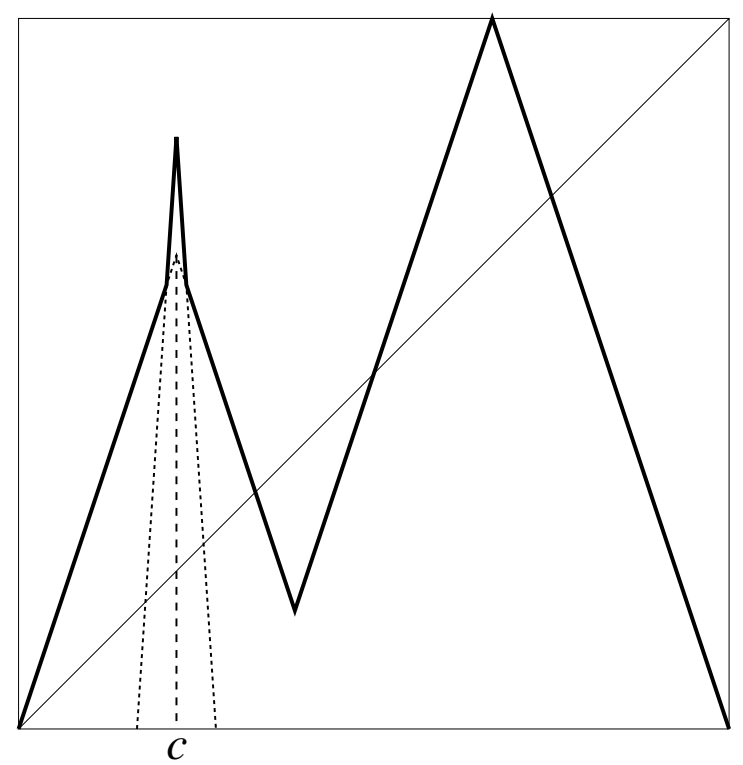

FiguRE 3. Inserting a needle.

that $\left|f^{i}(L)\right|<\varepsilon$ for $i=1, \ldots, n-1$, otherwise we replace $L$ by the image of $L$ under some iterate of $f$. If $L$ contains a lap of $f$, then $|L| \geq \varepsilon$ and $|f(L)| \geq \lambda \varepsilon>\varepsilon$, a contradiction. Therefore $f$ is at most 2-to- 1 on $L$, so $|f(L)|>|L| / 2 \geq \varepsilon / 2$. If follows that $n \geq 2$ and $|f(L)|<\varepsilon$. Then we can apply (A) to get $\left|f^{k+1}(L)\right|>|L| \geq \varepsilon$. Therefore $k+1>n$, i.e. $n \leq k$. The map $f$ is Lipschitz continuous with constant $\lambda$, SO

$$
\varepsilon<\left|f^{k+1}(L)\right| \leq \lambda^{k+1-n}\left|f^{n}(L)\right| \leq \lambda^{k}\left|f^{n}(L)\right| .
$$

This contradiction proves (B).

Now we take $\eta>0$ such that $\eta \leq \varepsilon / \lambda^{k}$, the interval $[c-\eta / 2, c+\eta / 2]$ is contained in $U$, and $\eta<\lambda^{k-1} /\left(2(m+1)^{k-1}\right)$. Then we drive a "needle" of slope $2 / \eta$ through the graph of $f$ from below at $x=c$. That is, for each $t \in[0,1]$ we define the map $f_{t}$ by

$$
f_{t}(x)=\max \left(f(x), t-\frac{2|x-c|}{\eta}\right)
$$

(see Figure 3).

Clearly, the map $t \mapsto f_{t}$ is continuous. The set $K$ on which $f_{t}$ and $f$ differ is either an empty set or an interval centered at $c$ of length smaller than $\eta$. Since $\eta<\varepsilon$ and the laps of $f$ have length at least $\varepsilon, f_{t}$ is a piecewise monotone map with the same modality as $f$. On some interval with the left endpoint $0, f$ and $f_{t}$ coincide, so $f$ and $f_{n}$ are simultaneously increasing or decreasing on the first lap. Moreover, $K \subset U$. Observe also that since the laps of $f$ are longer than $\eta$, the slope $2 / \eta$ of the "needle" is larger than $\lambda$.

In the case when $c$ is an endpoint of $I$ and $f$ has a local maximum at $c$, we use the same formula for $f_{t}$, but its graph looks like a half (left or right) of the needle. The interval $[c-\eta / 2, c+\eta / 2]$ becomes $[0, \eta / 2]$ or $[1-\eta / 2,1]$. The point $c$ is an endpoint 
of $K$ rather than its center. If $c=0$ the argument proving that the maps $f$ and $f_{t}$ are simultaneously increasing or decreasing on the first lap becomes straightforward - it is decreasing for $f$ because $f$ has a local maximum at 0 , and it is decreasing for the needle by the formula. Otherwise everything is the same as in the case when $c$ is a turning point, and the rest of the proof needs no changes.

Fix $t \in[0,1]$. We will show that the map $f_{t}$ is transitive. If $f_{t}=f$ then there is nothing to prove, so assume that $f_{t} \neq f$.

(C) If an interval $L$ is not contained in $K$ then $f(L) \subset f_{t}(L)$. This follows from the fact that at the endpoints of $K$ the maps $f$ and $f_{t}$ coincide and on $K$ the slope of $f_{t}$ is larger than the slope of $f$ (look at Figure 3).

(D) For any interval $L$ of length less than $\varepsilon$ we have $\left|f_{t}^{k}(L)\right|>2|L|$. If none of the intervals $f_{t}^{i}(L), i=0,1, \ldots, k-1$, is contained in $K$, this follows from (C) (use induction) and (A). If one of those intervals, $f_{t}^{i}(L)$, is contained in $K$, then

$$
\frac{\left|f_{t}^{i+1}(L)\right|}{\left|f_{t}^{i}(L)\right|} \geq \frac{1}{2} \cdot \frac{2}{\eta}=\frac{1}{\eta}>\frac{2(m+1)^{k-1}}{\lambda^{k-1}} .
$$

For all $j \neq i$ the slope is at least $\lambda$ and the map is at most $(m+1)$-to- 1 (remember that the modality of $f_{t}$ is $m$ ), so

$$
\frac{\left|f_{t}^{j+1}(L)\right|}{\left|f_{t}^{j}(L)\right|} \geq \frac{\lambda}{m+1} .
$$

Multiplying all those inequalities, we get

$$
\frac{\left|f_{t}^{k}(L)\right|}{|L|}>\frac{2(m+1)^{k-1}}{\lambda^{k-1}} \cdot\left(\frac{\lambda}{m+1}\right)^{k-1}=2 .
$$

This proves (D).

(E) If $L$ is an interval of length at least $\varepsilon$ then $f^{n}(L) \subset f_{t}^{n}(L)$ for all $n$. We prove it by induction. Clearly, $f^{0}(L)=L=f_{t}^{0}(L)$. Assume now that $|L| \geq \varepsilon$ and suppose that $f^{n}(L) \subset f_{t}^{n}(L)$ for some $n$. By $(\mathrm{B}),\left|f^{n}(L)\right|>\varepsilon / \lambda^{k}$, so $\left|f_{t}^{n}(L)\right|>\varepsilon / \lambda^{k}$. Since by the definition $\eta \leq \varepsilon / \lambda^{k}$, we get $\left|f_{t}^{n}(L)\right|>\eta$. Since $|K|<\eta$, the interval $f_{t}^{n}(L)$ is not contained in $K$. Then, by $(\mathrm{C})$ and the induction hypothesis,

$$
f^{n+1}(L)=f\left(f^{n}(L)\right) \subset f\left(f_{t}^{n}(L)\right) \subset f_{t}\left(f_{t}^{n}(L)\right)=f_{t}^{n+1}(L) .
$$

This completes the induction step.

Now we can finish the proof of the lemma. To prove transitivity of $f_{t}$, take intervals $J, M \subset I$. We have to show that there is $n$ such that $f_{t}^{n}(J) \cap M \neq \emptyset$. By (D) used inductively, there is $i$ such that $\left|f_{t}^{i}(J)\right| \geq \varepsilon$. Since $f$ is transitive, there exists $j$ such that $f^{j}\left(f_{t}^{i}(J)\right) \cap M \neq \emptyset$. By $(\mathrm{E}), f^{j}\left(f_{t}^{i}(J)\right) \subset f_{t}^{j}\left(f_{t}^{i}(J)\right)$. Therefore, $f_{t}^{j+i}(J) \cap M \neq \emptyset$. Hence, $f_{t}$ is transitive. Together with what we already proved just after the definition of $f_{t}$, it follows that $f_{t} \in \mathcal{T}_{m}^{*}$.

Thus, $\left\{f_{t}: t \in[0,1]\right\}$ is an arc in $\mathcal{T}_{m}^{*}$. We have $f_{0}=f$ and for $g=f_{1}$ we get

$$
g(c)=\max \left(f(c), 1-\frac{2|c-c|}{\eta}\right)=1 .
$$


In what follows, a map $f \in \mathcal{T}_{n}$ is called an $(n+1)$-horseshoe if each of the $n+1$ laps of $f$ is mapped onto the whole interval $I$.

Lemma 3.5. There is an $(n+1)$-horseshoe with constant slope in each arcwise connected component of each space $\mathcal{T}_{n}^{*}$.

Proof. Let $f \in \mathcal{T}_{n}^{*}$. Using Lemma 3.4 we can move one by one images of all turning points and endpoints of $I$ to 0 and 1 , staying in the same arcwise connected component of $\mathcal{T}_{n}^{*}$. The resulting map is a transitive $(n+1)$-horseshoe, which by Theorem 3.3 is positively conjugate to a map with a constant slope. This map, since it is conjugate to an $(n+1)$-horseshoe, must be an $(n+1)$-horseshoe. By Lemma 3.2, it is still in the same arcwise connected component of $\mathcal{T}_{n}^{*}$.

Lemma 3.6. Each of the sets $\mathcal{T}_{n}^{*}$ is open in $\mathcal{T}_{n}$.

Proof. Let $f \in \mathcal{T}_{n}^{*}$. If $c$ is a turning point of $f$ and $f$ has a local maximum at $c$, then for a sufficiently small $\varepsilon$ we have $f(c \pm \varepsilon)<f(c)$. For this $\varepsilon$, the same inequality holds if we replace $f$ by a map $g \in \mathcal{T}_{n}$ sufficiently close to $f$. Thus, there is a turning point $c^{\prime} \in(c-\varepsilon, c+\varepsilon)$ of $g$, and $g$ has at $c^{\prime}$ a local maximum. The situation is analogous for a local minimum. Therefore, if $\varepsilon$ is sufficiently small and $g \in \mathcal{T}_{n}$ is sufficiently close to $f$, then, since $g$ has only $n$ turning points, their types (local maximum, local minimum) come in the same order as for $f$. This means that if $g \in \mathcal{T}_{n}$ is sufficiently close to $f$ then $g$ belongs to the same space $\mathcal{T}_{n}^{*}$ as $f$.

For given $n$, there are only two $(n+1)$-horseshoes with constant slope. Thus, from Lemmas 3.5 and 3.6 we get immediately the following result.

Theorem 3.7. Each space $\mathcal{T}_{n}$ has two connected components, namely $\mathcal{T}_{n}^{+}$and $\mathcal{T}_{n}^{-}$. Each of those components is arcwise connected.

Lemma 3.8. Let $f \in \mathcal{C}$ be a piecewise monotone map with a constant slope $\lambda>2$, for which the image of every lap (except perhaps the leftmost and the rightmost ones) is the whole $I$. Then $f$ is transitive.

Proof. We will show that $f$ is locally eventually onto (topologically exact). If an interval $L \subset I$ does not contain two turning points, then $|f(L)| /|L| \geq \lambda / 2$. Since $\lambda / 2>1$, by using this property inductively we see that for every interval $J \subset I$ there is $n$ such that the interval $f^{n}(J)$ contains two turning points. Thus, it contains a lap which is neither leftmost nor rightmost, so $f^{n+1}(J)=I$.

Note that in the above lemma we cannot replace the assumption $\lambda>2$ by $\lambda \geq 2$. Indeed, if $\lambda=2$ and $f$ is decreasing on the first lap, then $f(0)$ is a fixed point and the interval $[0, f(0)]$ is invariant.

Proposition 3.9. For every $n \geq 1$ and $*$, \# $\in\{+,-\}$ there is an arc in $\mathcal{T}_{n}^{*} \cup \mathcal{T}_{n+1}^{\#}$ joining $\mathcal{T}_{n}^{*}$ with $\mathcal{T}_{n+1}^{\#}$.

Proof. Suppose we want to connect $\mathcal{T}_{n}^{+}$with $\mathcal{T}_{n+1}^{+}$. Then we use the maps $f_{t}$ defined by

$$
f_{t}(x)= \begin{cases}t x-\lfloor t x\rfloor & \text { if }\lfloor t x\rfloor \text { is even } \\ 1-(t x-\lfloor t x\rfloor) & \text { if }\lfloor t x\rfloor \text { is odd }\end{cases}
$$




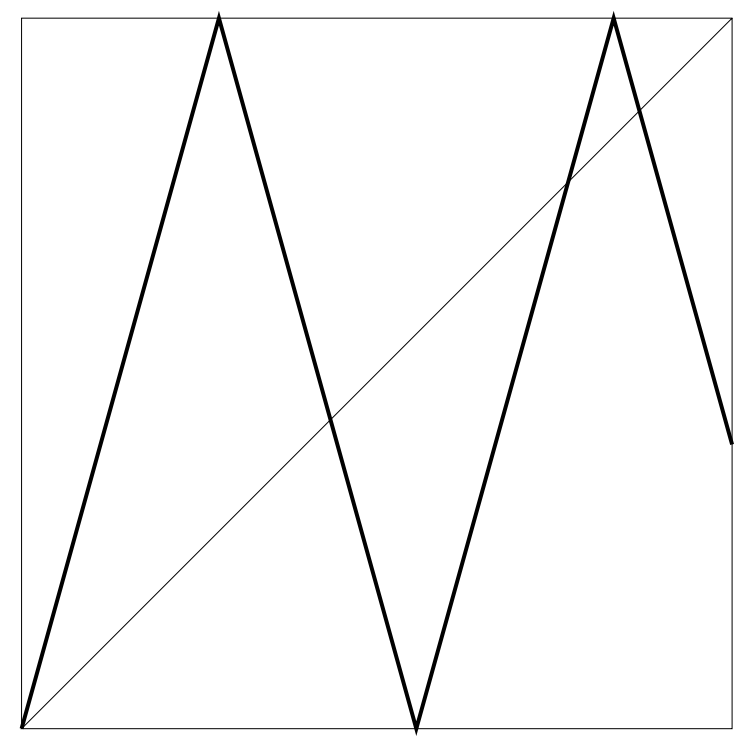

Figure 4. Map $f_{t}$, with $t=3.6$, from the proof of Proposition 3.9.

(see Figure 4). The map $f_{t}$ is a continuous map of constant slope $t$. By Lemma 3.8, if $t>2$ then $f_{t}$ is transitive. Additionally, $f_{2}$ is a 2-horseshoe, so it is also transitive. The map $f_{n+1}$ is an $(n+1)$-horseshoe of constant slope and belongs to $\mathcal{T}_{n}^{+}$; while the map $f_{n+2}$ is an $(n+2)$-horseshoe of constant slope and belongs to $\mathcal{T}_{n+1}^{+}$. All maps $f_{t}$ with $n+1<t \leq n+2$ have modality $n+1$. The map $t \mapsto f_{t}$ is clearly continuous, and hence the set $\left\{f_{t}: t \in[n+1, n+2]\right\}$ is an arc in $\mathcal{T}_{n}^{+} \cup \mathcal{T}_{n+1}^{+}$joining $\mathcal{T}_{n}^{+}$with $\mathcal{T}_{n+1}^{+}$.

For the other three arcs we use the families of maps $1-f_{t}, h_{t}$ given by $h_{t}(x)=$ $f_{t}(1-x)$, and $1-h_{t}$.

Remark 3.10. The arcs we constructed in the proof of the above proposition have some additional properties, that we will use later. Namely, each of them joins an $(n+1)$-horseshoe of constant slope to an $(n+2)$-horseshoe of constant slope and all points of the arc, except one endpoint, belong to $\mathcal{T}_{n+1}$.

As an immediate corollary to Theorem 3.7 and Proposition 3.9 we get the following result (it will be further generalized in Theorem 3.15).

Corollary 3.11. For every $n \geq 1$, the space $\mathcal{T}_{n} \cup \mathcal{T}_{n+1}$ is arcwise connected.

We investigate further our spaces.

Theorem 3.12. The set $\mathcal{T}_{n}$ is open in the space $\bigcup_{i=1}^{n} \mathcal{T}_{i}$.

Proof. As in the proof of Lemma 3.6, if $f \in \mathcal{T}_{n}$ and $g$ is sufficiently close to $f$ then in a small neighborhood of each turning point of $f$ there is a turning point of $g$. Thus, if $g \in \bigcup_{i=1}^{n} \mathcal{T}_{i}$ then $g \in \mathcal{T}_{n}$.

Remark 3.13. From Theorem 3.12 it follows that for every $n \geq k \geq 1$ the space $\bigcup_{i=k}^{n} \mathcal{T}_{i}$ is open in $\bigcup_{i=1}^{n} \mathcal{T}_{i}$.

To get more information about topology of (the unions of) the spaces we are considering, we generalize Proposition 3.9. 


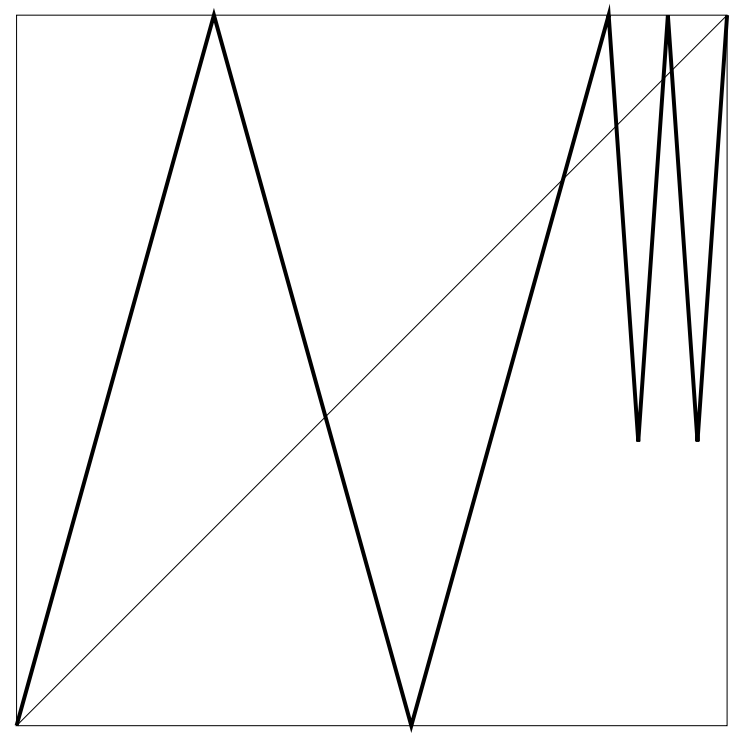

Figure 5. Map $g_{t}$, with $t=3.6, n=2$ and $m=6$ from the proof of Theorem 3.14.

Theorem 3.14. For every $m>n \geq 1$ and $*, \# \in\{+,-\}$ there is an arc in $\mathcal{T}_{m}^{*} \cup \mathcal{T}_{n}^{\#}$ joining $\mathcal{T}_{n}^{*}$ with $\mathcal{T}_{m}^{\#}$.

Proof. We modify the family of maps $f_{t}, t \in[n+1, n+2]$, from the proof of Proposition 3.9. Suppose first that $*=\#=+$. Then we replace the last lap of $f_{t}$, for $t \in(n+1, n+2]$, by $m-n$ laps of constant slope, each of them with the same image as the image of the last lap under $f_{t}$ (see Figure 5). A simple computation shows that this constant slope is $(m-n) t$. We call this modified map $g_{t}$.

Clearly, $g_{t}$ depends continuously on $t$ for $t \in[n+1, n+2]$ and is increasing on the first lap; $g_{n+1}$ is an $(n+1)$-horseshoe of constant slope, and all maps $g_{t}$ for $t \in(n+1, n+2]$ have modality $m$. It remains to prove that the maps $g_{t}$ for $t \in(n+1, n+2]$ are transitive.

Fix $t \in(n+1, n+2]$. We will show as in the proof of Lemma 3.8 that $g_{t}$ is locally eventually onto. If an interval $L \subset I$ does not contain two turning points, then $\left|g_{t}(L)\right| /|L| \geq t / 2$. Since $t / 2>1$, by using this property inductively we see that for every interval $J \subset I$ there is $k$ such that the interval $g_{t}^{k}(J)$ contains two turning points. If these are two turning points of the original map $f_{t}$, then $g_{t}^{k+1}(J)=I$ and we are done. If $g_{t}^{k}(J)$ does not contain such two turning points, then it contains two turning points of the part of the map $g_{t}$ which is different from $f_{t}$. Then $f_{t}\left(g_{t}^{k}(J)\right) \subset g_{t}\left(g_{t}^{k}(J)\right)$, and therefore $\left|g_{t}\left(g_{t}^{k}(J)\right)\right| /\left|g_{t}^{k}(J)\right| \geq t / 2$. Continuing this, we see that eventually some image of $J$ will contain two turning points of $f_{t}$, and the next image will be equal to $I$. This proves that $g_{t}$ is locally eventually onto, and therefore transitive.

For the remaining choices of $*$, \# we can use, as in the proof of Proposition 3.9 the families of maps $1-g_{t}, h_{t}$ given by $h_{t}(x)=g_{t}(1-x)$, and $1-g_{t}$, except when $m-n$ is even and $* \neq \#$. In this special case on both first and last laps of the elements of $\mathcal{T}_{n}^{*}$ the type of monotonicity is different than for the elements of $\mathcal{T}_{m}^{\#}$. Then we have 
to start not from the family $f_{t}$, but from the family $\widetilde{f}_{t}$, given by

$$
\tilde{f}_{t}(x)= \begin{cases}t x-\frac{t-(n+1)}{2}-\left\lfloor t x-\frac{t-(n+1)}{2}\right\rfloor & \text { if }\left\lfloor t x-\frac{t-(n+1)}{2}\right\rfloor \text { is even, } \\ 1-\left(t x-\frac{t-(n+1)}{2}-\left\lfloor t x-\frac{t-(n+1)}{2}\right\rfloor\right) & \text { if }\left\lfloor t x-\frac{t-(n+1)}{2}\right\rfloor \text { is odd }\end{cases}
$$

For $t=n+1$ this is $(n+1)$-horseshoe increasing on the first lap, but for $t \in(n+1, n+3]$ it has $n+3$ laps and is decreasing on the first one. If $m=n+2$, choose the families of maps $\tilde{f}_{t}$ or $1-\widetilde{f}_{t}, t \in[n+1, n+2]$, to produce arcs joining $\mathcal{T}_{n}^{+}$with $\mathcal{T}_{m}^{-}$or $\mathcal{T}_{n}^{-}$ with $\mathcal{T}_{m}^{+}$, respectively. If $m-n>2$ is even, we make the same type of modifications as in the current proof to get $g_{t}$, and we make them only on the last lap. Again the proof goes through with just minor changes.

As a corollary, we get immediately the following result.

Theorem 3.15. For every $m>n \geq 1$, the space $\mathcal{T}_{n} \cup \mathcal{T}_{m}$ is arcwise connected. In fact, the space $\bigcup_{i \in A} \mathcal{T}_{i}^{*(i)}$ is arcwise connected, whenever $\mathcal{T}_{i}^{*(i)} \in\left\{\mathcal{T}_{i}^{+}, \mathcal{T}_{i}^{-}, \mathcal{T}_{i}\right\}$ and $A$ is a set of positive integers of cardinality at least 2.

Remark 3.16. Similarly as in Remark 3.10, the arcs in the prof of Theorem 3.14 are such that only one endpoint is in $\mathcal{T}_{n}^{\#}$; all the other points of the arc are in $\mathcal{T}_{m}^{*}$. Taking into account that each map from $\mathcal{T}_{m}$ has a neighborhood such that all (piecewise monotone) maps in this neighborhood are at least $m$-modal (cf. Theorem 3.12), we get that $\mathcal{T}_{n}^{\#}$ is nowhere dense and closed in $\mathcal{T}_{m}^{*} \cup \mathcal{T}_{n}^{\#}$ and also nowhere dense and closed in $\mathcal{T}_{m} \cup \mathcal{T}_{n}^{\#}$, whenever $m>n \geq 1$.

Now we compute the distance between $\mathcal{T}_{1}^{+}$and $\mathcal{T}_{1}^{-}$.

Theorem 3.17. The distance between $\mathcal{T}_{1}^{+}$and $\mathcal{T}_{1}^{-}$, that is, $\inf \left\{d(f, g): f \in \mathcal{T}_{1}^{+}, g \in\right.$ $\left.\mathcal{T}_{1}^{-}\right\}$is $1 / 3$. Moreover, the infimum is not attained.

Proof. Let $f \in \mathcal{T}_{1}^{+}$and $g \in \mathcal{T}_{1}^{-}$. We want to show first that $d(f, g)>1 / 3$. Remember that the maps from $\mathcal{T}_{1}$ are unimodal. Denote the turning points of $f$ and $g$ by $c_{f}$ and $c_{g}$ respectively. Each map from $\mathcal{T}_{1}$ has exactly one fixed point in $(0,1)$; denote those fixed points of $f$ and $g$ by $a_{f}$ and $a_{g}$ respectively.

Since $f$ is transitive, $a_{f}$ has two preimages. To see it, observe that since $f$ is conjugate to a map with constant slope larger than 1 , the fixed point $a_{f}$ is repelling. Thus, if $a_{f}$ has only one preimage, then the set $\left[0, f^{2}(0)\right] \cup[f(0), 1]$ is invariant, a contradiction. Similarly, $a_{g}$ has two preimages under $g$. Therefore, $f(0) \leq a_{f}$ and $g(1) \geq a_{g}$. Moreover, $f\left(c_{f}\right)=1, f(1)=0, g\left(c_{g}\right)=0$ and $g(0)=1$ (see Figure 6).

We have

$$
d(f, g) \geq \max (|f(0)-g(0)|,|f(1)-g(1)|)=\max (1-f(0), g(1)) \geq \max \left(1-a_{f}, a_{g}\right) \text {. }
$$

If $a_{f} \leq a_{g}$, we get

$$
d(f, g) \geq \max \left(1-a_{f}, a_{f}\right) \geq \frac{1}{2}>\frac{1}{3} .
$$

Assume that $a_{f}>a_{g}$ and $d(f, g) \leq 1 / 3$. In particular, we have $f(0) \geq 2 / 3$ because $g(0)=1$. Then $f\left(a_{f}\right)=a_{f} \geq f(0) \geq 2 / 3$, so $f(x)>2 / 3$ for all $x \in\left(0, a_{f}\right)$. Similarly, $g(x)<1 / 3$ for all $x \in\left(a_{g}, 1\right)$. Hence, $f(x)-g(x)>2 / 3-1 / 3=1 / 3$ for all $x \in\left(a_{g}, a_{f}\right)$, so $d(f, g)>1 / 3$. 


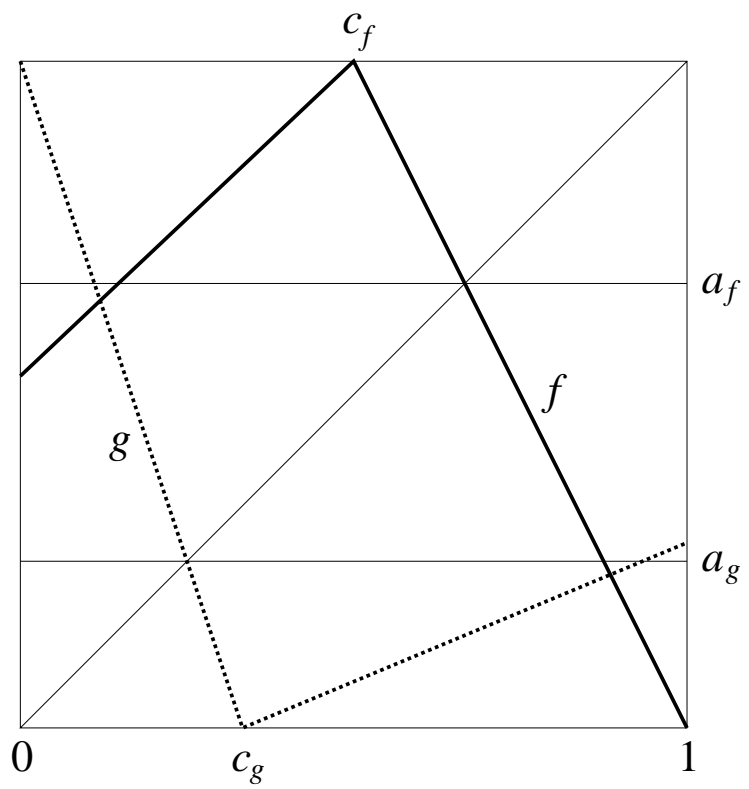

Figure 6. Maps $f \in \mathcal{T}_{1}^{+}$and $g \in \mathcal{T}_{1}^{-}$.

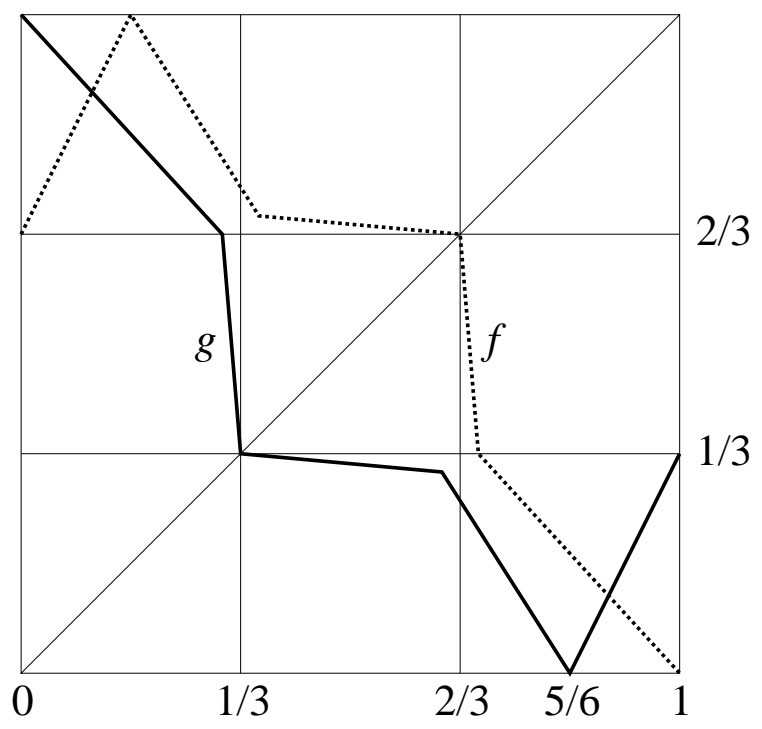

Figure 7. Maps $f$ and $g$ from the second part of the proof of Theorem 3.17.

Now we will construct maps $f \in \mathcal{T}_{1}^{+}$and $g \in \mathcal{T}_{1}^{-}$with $d(f, g)$ close to $1 / 3$. Fix $\varepsilon \in(0,1 / 6)$. The map $g$ will be a piecewise linear "connect the dots" map with the dots at $(0,1),(1 / 3-\varepsilon, 2 / 3),(1 / 3,1 / 3),(2 / 3-\varepsilon, 1 / 3-\varepsilon),(5 / 6,0)$ and $(1,1 / 3)$ (see Figure 7). The graph of $f$ will be symmetric to the graph of $g$ with the center of symmetry at $(1 / 2,1 / 2)$, that is, $f$ will be conjugate to $g$ via $x \mapsto 1-x$.

Let us prove that $g$ is transitive. We have $g([0,1 / 3])=[1 / 3,1]$ and $g([1 / 3,1])=$ $[0,1 / 3]$. Therefore, it is enough to show that $g^{2}$ restricted to $[1 / 3,1]$ is transitive. 
Elementary computations show that $\left.g^{2}\right|_{[1 / 3,1]}$ is a piecewise linear "connect the dots" map with the dots at $(1 / 3,1 / 3),(2 / 3-\varepsilon, 2 / 3),(5 / 6,1),(1-\varepsilon / 2,2 / 3)$ and $(1,1 / 3)$. Thus, it is a 2-horseshoe, piecewise linear with the minimal slope $\lambda=1 /(1-3 \varepsilon)>1$. Such a map is always transitive. Indeed, the lengths of the consecutive intervals grow at least by the factor $\lambda$, until they hit the turning point. Then, after two iterates, they cover an interval of the form $[1 / 3,1 / 3+\delta]$ for some $\delta>0$. After some more iterates, we get the interval $[1 / 3,1]$, so $\left.g^{2}\right|_{[1 / 3,1]}$ is transitive.

Thus, we proved that $g$ is transitive. The map $f$ is conjugate to $g$, so it is also transitive. Hence, $f \in \mathcal{T}_{1}^{+}$and $g \in \mathcal{T}_{1}^{-}$.

From the definitions of $f$ and $g$ (see Figure 7 ), it is clear that $d(f, g) \rightarrow 1 / 3$ as $\varepsilon \rightarrow 0$. This completes the proof.

Remark 3.18. In the proof of Proposition 3.9 we constructed maps from both $\mathcal{T}_{n+1}^{+}$ and $\mathcal{T}_{n+1}^{-}$arbitrarily close to each of $(n+1)$-horseshoes of constant slope (see Remark 3.10). Therefore the distance between $f \in \mathcal{T}_{n}^{+}$and $g \in \mathcal{T}_{n}^{-}$is zero for all $n \geq 2$.

Let us try to get some information about the fundamental group of (the unions of) the spaces we are investigating.

Theorem 3.19. For every $n \geq 1$ there is a loop in $\mathcal{T}_{n} \cup \mathcal{T}_{n+1}$, which is not contractible in $\mathcal{T}_{n} \cup \mathcal{T}_{n+1}$.

Proof. Let us take a loop $L$ that is a concatenation, in a proper order, of the four arcs constructed in the proof of Proposition 3.9. By Remark 3.10 it is indeed a loop and only two points in it belong to $\mathcal{T}_{n}$; one of them to $\mathcal{T}_{n}^{+}$and the other one to $\mathcal{T}_{n}^{-}$. Two arcs (without the endpoints) into which those points divide the loop are contained: one in $\mathcal{T}_{n+1}^{+}$and the other one in $\mathcal{T}_{n+1}^{-}$.

Suppose that this loop is contractible in $\mathcal{T}_{n} \cup \mathcal{T}_{n+1}$. Then there is a continuous map $\varphi: \Delta \rightarrow \mathcal{T}_{n} \cup \mathcal{T}_{n+1}$, where

$$
\Delta=\left\{(x, y): x^{2}+y^{2} \leq 1\right\}
$$

is the unit disk, such that the boundary of $\Delta$ is mapped homeomorphically onto $L$.

By Theorem 3.12 and Lemma 3.6, the sets $\varphi^{-1}\left(\mathcal{T}_{n+1}^{+}\right)$and $\varphi^{-1}\left(\mathcal{T}_{n+1}^{-}\right)$are open in $\Delta$. Therefore the set $\varphi^{-1}\left(\mathcal{T}_{n}\right)$ is closed in $\Delta$. Moreover, by Lemma 3.6 the sets $\mathcal{T}_{n}^{+}$ and $\mathcal{T}_{n}^{-}$are closed in $\mathcal{T}_{n}$, so $\varphi^{-1}\left(\mathcal{T}_{n}^{+}\right)$and $\varphi^{-1}\left(\mathcal{T}_{n}^{-}\right)$are closed in $\Delta$.

We get the following picture. The disk is partitioned into four sets, $A=\varphi^{-1}\left(\mathcal{T}_{n+1}^{+}\right)$, $B=\varphi^{-1}\left(\mathcal{T}_{n+1}^{-}\right), C=\varphi^{-1}\left(\mathcal{T}_{n}^{+}\right)$, and $D=\varphi^{-1}\left(\mathcal{T}_{n}^{-}\right)$. The sets $A$ and $B$ are open in $\Delta$, and $C$ and $D$ are closed in $\Delta$ (and therefore compact). The intersection of $C$ with the boundary $\partial \Delta$ of $\Delta$ consists of one point, call it $p$. Similarly, the intersection of $D$ with $\partial \Delta$ consists of one point, call it $q$. The set $\partial \Delta \backslash\{p, q\}$ is the union of two open arcs; one of them is contained in $A$, and the other one in $B$.

We will show that this leads to a contradiction. We may assume that $p=(0,1)$ and $q=(0,-1)$. We compactify the plane with one point $\infty$ and get (topologically) a sphere $\mathbb{S}^{2}$. Let $Y$ be the complement in $\mathbb{S}^{2}$ of the rectangle $(-2,2) \times(-1,1)$ (see Figure 8). Points $r=(-1,0)$ and $s=(1,0)$ can be joined by an arc in $\mathbb{S}^{2} \backslash(Y \cup$ $D)$. Indeed, we can go from $r$ along $\partial \Delta$ almost to $p$, then go around $p$ in a small neighborhood of $p$ in $\Delta$, and then continue to $s$ along $\partial \Delta$ (see Figure 8). If this neighborhood of $p$ is sufficiently small, then it is disjoint from $D$, because $p \in C$ 


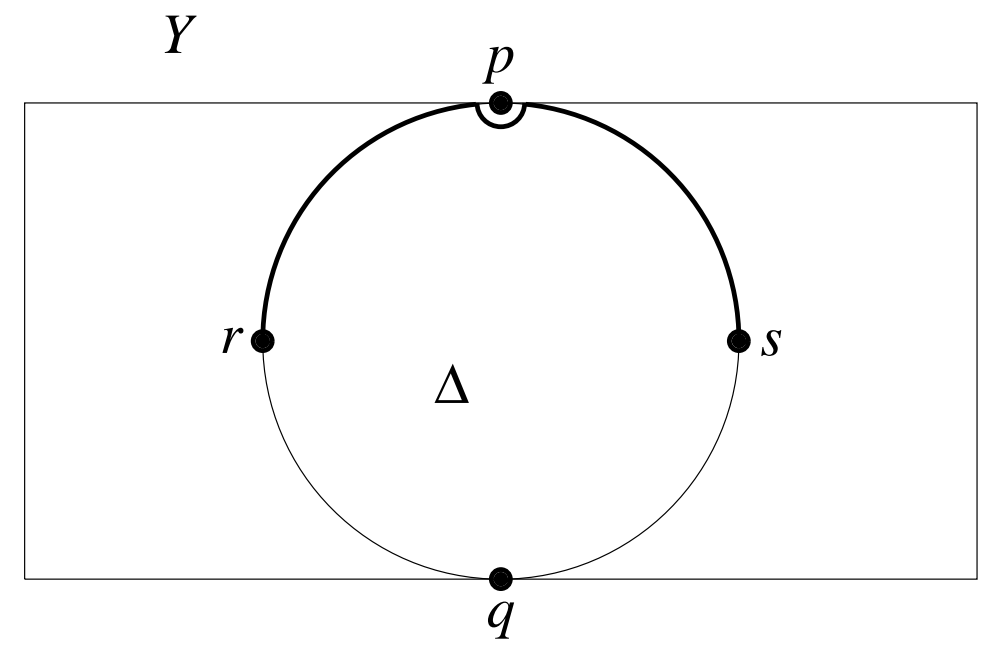

Figure 8. Topological construction in the one-point compactification of the plane from the proof of Theorem 3.19.

and $C$ and $D$ are disjoint compact sets. Thus, the whole arc is disjoint from $D$. Similarly, $r$ and $s$ can be joined by an arc in $\mathbb{S}^{2} \backslash(Y \cup C)$. Since $Y \cup D$ and $Y \cup C$ are closed subsets of $\mathbb{S}^{2}$, none of them being a cut between $r$ and $s$, and their intersection $(Y \cup D) \cap(Y \cup C)=Y$ is connected, we can use [5, Theorem 7, §61, p. 507], which says that in this situation $(Y \cup D) \cup(Y \cup C)$ is not a cut between $r$ and $s$. So, there is a continuum in $\mathbb{S}^{2} \backslash((Y \cup D) \cup(Y \cup C))$ joining $r$ with $s$.

The retraction of this continuum to $\Delta$ along the rays from the origin is also a continuum joining $r$ with $s$, and it is contained in $A \cup B$. This is a contradiction, because by Theorem 3.7 the points $r$ and $s$ belong to different connected components of $\varphi^{-1}\left(\mathcal{T}_{n+1}\right)$. This completes the proof.

\section{REFERENCES}

[1] Ll. Alsedà, J. Llibre and M. Misiurewicz, Combinatorial Dynamics and Entropy in Dimension One, 2nd edition, Advanced Series in Nonlinear Dynamics 5, World Scientific, Singapore, 2000.

[2] F. T. Farrell and A. Gogolev, The space of Anosov diffeomorphisms, arXiv:1201.3595v1 [math.DS].

[3] J. G. Hocking and G. S. Young, Topology, 2nd edition, Dover Publications, Inc., New York, 1988.

[4] S. Kolyada and L. Snoha, Topological transitivity, Scholarpedia, 4(2):5802 (2009), http://www. scholarpedia.org/article/Topological_transitivity.

[5] K. Kuratowski, Topology, Vol. II, Academic Press, New York-London, Państwowe Wydawnictwo Naukowe ((Polish Scientific Publishers), Warsaw, 1968.

[6] J. Milnor and W. Thurston, On iterated maps of the interval, in "Dynamical systems (College Park, MD, 1986-87)," Lecture Notes in Math. 1342, Springer, Berlin, 1988, pp. 465-563.

[7] W. Parry, Symbolic dynamics and transformations of the unit interval, Trans. Amer. Math. Soc. 122 (1966), 368-378.

[8] G. T. Whyburn, Analytic topology, AMS Coll. Publications 28, Providence, RI, 1942. 
Institute of Mathematics, NASU, Tereshchenkivs'Ka 3, 01601 Kiev, Ukraine

E-mail address: skolyada@imath.kiev.ua

Department of Mathematical Sciences, Indiana University - Purdue University Indianapolis, 402 N. Blackford Street, Indianapolis, IN 46202, USA

E-mail address: mmisiure@math.iupui.edu

Department of Mathematics, Faculty of Natural Sciences, Matej Bel University, TAjovskéHo 40, 97401 Banská Bystrica, Slovakia

E-mail address: Lubomir.Snoha@umb.sk 